\title{
Juxtacortical Lesions and Cortical Thinning in Multiple Sclerosis
}

\author{
D. Pareto, J. Sastre-Garriga, C. Auger, Y. Vives-Gilabert, J. Delgado, M. Tintoré, X. Montalban, and A. Rovira
}

\begin{abstract}
BACKGROUND AND PURPOSE: The role of juxtacortical lesions in brain volume loss in multiple sclerosis has not been fully clarified. The aim of this study was to explore the role of juxtacortical lesions on cortical atrophy and to investigate whether the presence of juxtacortical lesions is related to local cortical thinning in the early stages of MS.
\end{abstract}

MATERIALS AND METHODS: A total of 131 patients with clinically isolated syndrome or with relapsing-remitting MS were scanned on a $3 T$ system. Patients with clinically isolated syndrome were classified into 3 groups based on the presence and topography of brain lesions: no lesions ( $n=24)$, only non-juxtacortical lesions ( $n=33$ ), and juxtacortical lesions and non-juxtacortical lesions $(n=34)$. Patients with relapsing-remitting MS were classified into 2 groups: only non-juxtacortical lesions $(n=10)$ and with non-juxtacortical lesions and juxtacortical lesions $(n=30)$. A juxtacortical lesion probability map was generated, and cortical thickness was measured by using FreeSurfer.

RESULTS: Juxtacortical lesion volume in relapsing-remitting MS was double that of patients with clinically isolated syndrome. The insula showed the highest density of juxtacortical lesions, followed by the temporal, parietal, frontal, and occipital lobes. Patients with relapsingremitting MS with juxtacortical lesions showed significantly thinner cortices overall and in the parietal and temporal lobes compared with those with clinically isolated syndrome with normal brain MR imaging. The volume of subcortical structures (thalamus, pallidum, putamen, and accumbens) was significantly decreased in relapsing-remitting MS with juxtacortical lesions compared with clinically isolated syndrome with normal brain MR imaging. The spatial distribution of juxtacortical lesions was not found to overlap with areas of cortical thinning.

CONCLUSIONS: Cortical thinning and subcortical gray matter volume loss in patients with a clinically isolated syndrome or relapsingremitting MS was related to the presence of juxtacortical lesions, though the cortical areas with the most marked thinning did not correspond to those with the largest number of juxtacortical lesions.

ABBREVIATIONS: $\mathrm{CIS}=$ clinically isolated syndrome; $\mathrm{CISj}=\mathrm{CIS}$ with juxtacortical lesion; $\mathrm{CISn}=\mathrm{CIS}$ with normal brain $\mathrm{MR}$ imaging; $\mathrm{CISnj}=\mathrm{CIS}$ without juxtacortical lesion; $\mathrm{L}$ = juxtacortical lesion; $\mathrm{JLV}$ = juxtacortical lesion volume; $\mathrm{LV}$ = lesion volume; $\mathrm{RRj}$ = relapsing-remitting MS with JL; RRMS = relapsing-remitting $M S ; R R j=$ relapsing-remitting MS without $\mathrm{L}$

M ultiple sclerosis is a chronic, persistent inflammatory-demyelinating disease of the central nervous system, characterized pathologically by focal areas of inflammation, demyelination, axonal loss, and gliosis. Brain MR imaging typically shows multifocal lesions, mainly in white matter regions, ${ }^{1}$

Received March 10, 2015; accepted after revision May 8.

From Unitat de Ressonància Magnètica (IDI), Servei de Radiologia (D.P., C.A., A.R.), Servei de Neurologia/Neuroimmunologia, Multiple Sclerosis Centre of Catalonia (Cemcat) (J.S.-G., M.T., X.M.), Hospital Universitari Vall d'Hebron, and Port d'Informació Científica (Y.V.-G., J.D.), Universitat Autònoma de Barcelona, Barcelona, Spain.

Please address correspondence to Àlex Rovira, MD, Unitat de Ressonància Magnètica (IDI), Servei de Radiologia, Hospital Universitari Vall d'Hebron, Passeig Vall d'Hebron 119-129, 08035 Barcelona, Spain; e-mail: alex.rovira@idi.gencat.cat

http://dx.doi.org/10.3174/ajnr.A4485 though focal cortical demyelinated plaques are also a prominent feature, even in the earliest phases of the disease. ${ }^{2}$ Unfortunately, conventional MR imaging has limited sensitivity for detecting cortical lesions because of their small size, the poor contrast resolution, and the partial volume effects of the subarachnoid spaces and surrounding cortex. ${ }^{3,4}$ Thus, histopathologic studies are the only way to describe, quantify, and classify gray matter lesions according to their position in relation to the gray-white matter surface (leukocortical or juxtacortical; intracortical and subpial). ${ }^{5,6}$ Despite the limited sensitivity of MR imaging for detecting cortical lesions in MS, results of several studies showed that cross-sectional cortical lesion volume and its increase over time are associated with progression of disability and cognitive impairment in MS..$^{7-10}$ 
Table 1: Demographic and clinical features of the study sample

\begin{tabular}{lccccc}
\hline & CISn & CISnj & CISj & RRnj & RRj \\
\hline$N$ (women) & $24(15)$ & $33(20)$ & $34(20)$ & $10(4)$ & $30(20)$ \\
Mean (SD) age, y & $36 \pm 10$ & $36 \pm 7$ & $35 \pm 8$ & $34 \pm 7$ & $36 \pm 7$ \\
Mean (SD) EDSS & $1.4 \pm 0.9$ & $1.4 \pm 0.9$ & $1.9 \pm 1.1$ & $3.2 \pm 2.0$ & $2.5 \pm 1.6$ \\
Mean (SD) DIS & $3-5$ mo & $3-5$ mo & $3-5$ mo & $10.4 \pm 8.2 y$ & $10.6 \pm 7.1 \mathrm{y}$ \\
LV mL (SD) & 0 & $0.75 \pm 1.17$ & $4.10 \pm 6.38$ & $3.12 \pm 3.71$ & $9.10 \pm 7.86$ \\
\hline
\end{tabular}

Note:-EDSS indicates expanded disability scale; DIS, disease duration.

Brain atrophy, which is also frequently detected by MR from the earliest stages of MS, is associated with irreversible neurologic disability, including cognitive impairment. ${ }^{11-14}$ Whole-brain atrophy has emerged as a clinically relevant component of disease progression, and results of several studies showed that this parameter correlates better with disability and, in particular, with cognitive impairment than with focal lesions. ${ }^{15}$ Although most brain atrophy measurements are based on global or regional (gray and white matter) brain volume assessment, cortical thickness has recently emerged as a new way to assess cortical gray matter atrophy because decreased thickness is related to fatigue, disability in general, and cognitive impairment in particular. ${ }^{13,16}$ This measurement seems to be dependent on focal white matter lesion volume, ${ }^{17}$ but a potential relationship between the presence and location of demyelinating juxtacortical lesions (JLs) and cortical atrophy has not been elucidated. Therefore, the aim of this study was to explore the role of JLs on cortical atrophy and to investigate whether their presence is related to local cortical thinning in the early stages of MS.

\section{MATERIALS AND METHODS}

\section{Patients}

A sample of 91 consecutive patients with a clinically isolated syndrome (CIS) and 40 patients with relapsing-remitting MS (RRMS) who were undergoing brain MR imaging at Vall d'Hebron University Hospital for diagnostic purposes or monitoring disease evolution were included in the study. CIS is a clinical description, and many lesions may exist on MR imaging in these patients. ${ }^{18}$ Patients with CIS were classified into 3 groups according to the presence of JLs and other typical demyelinating lesions on brain MR examination: no lesions (CISn, $n=24$ ), only non-JLs (CISnj, $n=33$ ), and with JLs plus non-JLs (CISj, $n=34$ ). Patients with RRMS were divided into 2 groups (none of the patients with RRMS had normal MR images): with only non-JLs (RRnj, $n=10$ ) and with JLs plus non-JLs (RRj, $n=30$ ). None of the patients had only JLs in the absence of other typical MS lesions on MR imaging. The characteristics of the 5 groups are summarized in Table 1. The study was approved by the local ethics committee. Because the study was based on MR imaging data acquired in regular clinical practice, the need for written informed consent from the participants was waived.

\section{MR Image Acquisition}

Images were acquired on a 3T whole-body MR scanner (Tim Trio, Siemens; Erlangen, Germany) with a 12-channel phased-array head coil and a whole-body transmit coil. The standard MS protocol included (besides other sequences): 1) fast dual-echo T2weighted transverse sequence $\left(\mathrm{TR}=3080\right.$ milliseconds, $\mathrm{TE}_{1}=21$ milliseconds, $\mathrm{TE}_{2}=91$ milliseconds, voxel size $=0.78 \times 0.78 \times$ $\left.3.0 \mathrm{~mm}^{3}\right) ; 2$ ) transverse T2-FLAIR sequence ( $\mathrm{TR}=9000$ milliseconds, $\mathrm{TE}=87$ milliseconds, $\mathrm{TI}=2500$ milliseconds, voxel size $=$ $\left.0.49 \times 0.49 \times 3.0 \mathrm{~mm}^{3}\right)$; and 3) sagittal 3D T1-weighted gradient-echo (MPRAGE) sequence $(\mathrm{TR}=2300$ milliseconds, $\mathrm{TE}=$ 3000 milliseconds, voxel size $=1.0 \times$ $1.0 \times 1.2 \mathrm{~mm}^{3}$ ). Total scanning time for these sequences was 15 minutes.

\section{Image Analysis}

All supratentorial JLs were identified and manually delineated on T2-FLAIR images by using Jim software (http://www.xinapse.com/home.php). JL volume (JLV) was also calculated. To obtain JL probability maps, T2-FLAIR images and the corresponding lesion masks were normalized to the Montreal Neurological Institute template by using Statistical Parametric Mapping software (SPM8; Wellcome Department of Imaging Neuroscience, London, U.K.; http://www.fil.ion.ucl.ac.uk/spm/ software/spm8). Once images and masks were spatially normalized, a mean image, which represents the JL probability map, was generated for the CIS and RRMS groups. A ROI for each brain lobe was delineated, based on the Automatic Anatomical Labeling set of ROIs. ${ }^{19}$ The final ROI included gray and white matter. The percentage of JLs in each brain lobe relative to the total number of JLs in the whole brain was calculated and normalized to the ROI volume. White matter lesion volume (LV) was estimated for each patient by using the automated Lesion Segmentation Toolbox, ${ }^{20}$ which obtains lesion masks and associated total LVs based on the T2-FLAIR and 3D T1-weighted images. Toolbox parameters had been optimized previously for the 2D T2-FLAIR images included in this cohort. ${ }^{21}$ Cortical thickness was measured in single time points with MPRAGE images by using the FreeSurfer program suite (version 5.1; http://surfer.nmr.mgh.harvard.edu/). ${ }^{10}$ Briefly, white matter points are chosen based on their locations in the Talairach space as well as on their intensity and the local neighborhood intensities. Voxels are then classified as white matter or something other than white matter based on intensity and neighbor constraints. Cutting planes are chosen to separate the hemispheres from each other. An initial surface is then generated for each hemisphere by tiling the outside of the white matter mass for that hemisphere. This initial surface is then refined to follow the intensity gradients between the white and gray matter (this is referred to as the white surface). The white surface is then nudged to follow the intensity gradients between the gray matter and CSF (this is the pial surface). The distance between the white and the pial surface gives us the thickness at each location of cortex. ${ }^{22,23}$ Subcortical gray matter volume measurements were also obtained as part of the established pipeline. ${ }^{22,23}$ Estimated subcortical gray matter volumes were multiplied by 100 and divided by the corresponding estimated total intracranial volume obtained for each subject. Finally, the FreeSurfer output segmentations were carefully reviewed, particularly checking for accuracy at the sites where JLs occurred. Cortical thickness was estimated in the whole brain, lobes, and regions, and at the vertex level in each patient. The mean cortical thickness value for each lobe was the average of values obtained for various FreeSurfer parcellations as followsfrontal lobe: caudal anterior cingulate, caudal middle frontal, isthmus cingulate, lateral orbitofrontal, medial orbitofrontal, paracentral, pars opercularis, pars orbitalis, pars triangularis, pre- 
central, rostral anterior cingulate, superior frontal, and frontal pole; parietal lobe: inferior parietal, pericalcarine, postcentral, posterior cingulate, precuneus, and supramarginal; temporal lobe: banks of superior temporal sulcus, fusiform, entorhinal, inferior temporal, middle temporal, parahippocampal, superior temporal, temporal pole, and transverse temporal; and occipital lobe: cuneus, lateral occipital, and lingual; and insula.

\section{Statistical Analysis}

Whole white matter LV and JLV and distribution were compared across groups by means of 1-way ANOVA by using the factor group, followed by pair-wise Bonferroni post hoc comparison to account for multiple comparisons.

Differences in cortical thickness between the groups were assessed at 3 different levels: by using the global values; by using values after the parcellation process; and, at the vertex level, by using the cortical thickness map. First, differences between the left and right hemispheres were tested by 1-way ANOVA by using the factor hemisphere, followed by post hoc Bonferroni comparison. Then, the cortical thickness values obtained were compared across groups by 1-way ANOVA, followed by pair-wise Bonferroni post hoc comparison. As to the vertex level comparison, statistical difference maps (between patients with CISnj and patients with CISj, and RRnj and RRj) were generated based on general linear model analysis by using the FreeSurfer QDEC tool.

In the estimation of subcortical gray matter volumes, the right-left difference was assessed by 1-way ANOVA with the factor hemisphere. Differences in subcortical gray matter volumes

Table 2: Distribution of JLs ${ }^{\mathrm{a}}$

\begin{tabular}{|c|c|c|c|c|}
\hline Lobe & $\begin{array}{l}\text { LPMvol CIS, } \\
\text { no. (\%) }\end{array}$ & $\begin{array}{c}\text { LPMp CIS, } \\
\%\end{array}$ & $\begin{array}{c}\text { LPMvol RRMS, } \\
\text { no. (\%) }\end{array}$ & $\begin{array}{c}\text { LPMp RRMS, } \\
\%\end{array}$ \\
\hline Frontal & $11.6(39.3)$ & 1.7 & $27.0(46)$ & 4.0 \\
\hline Insula & $1.3(4.5)$ & 5.0 & $1.9(3.2)$ & 7.0 \\
\hline Parietal & $7.7(26.2)$ & 2.0 & $14.2(24.3)$ & 3.7 \\
\hline Temporal & 7.7 (26.12) & 2.6 & $13.9(23.8)$ & 4.7 \\
\hline Occipital & $1.1(3.9)$ & 0.7 & $1.6(2.8)$ & 0.9 \\
\hline
\end{tabular}

Note:- LPM indicates lesion probability map; LPMvol, absolute volume in millititers of $J$ per lobe (percentage of global $J \mathrm{~L}$ volume); LPM $\rho$, percentage of $J \mathrm{~L}$ volume relative to lobe volume.

a Total JLV (LPMvol) in milliliters (percentage relative to global amount), and percentage relative to lobe volume in patients with CIS and patients with RRMS. were compared across groups by 1-way ANOVA with the factor group, followed by pair-wise Bonferroni post hoc comparison.

Finally, to assess which disease burden-related parameter (presence of JL, JLV, or LV) was more relevant in the cortical thickness measurements, 3 multivariate models were tested by using the presence of JL, JLV, or white matter LV, as independent variables. The 3 models included age and sex as covariates. Significance was set at $P<.05$.

\section{RESULTS}

\section{Whole White Matter Lesion Volume}

The mean LV in the various patient groups is reported in Table 1. The rank order was CISnj $<\mathrm{RRnj}<\mathrm{CISj}<\mathrm{RRj}$. The betweengroup differences in LV were significant $(P<.001)$, and statistically significant differences were observed for the following post hoc comparisons $(P<.002): \mathrm{RRj}>\mathrm{RRnj}, \mathrm{RRj}>\mathrm{CISj}, \mathrm{RRj}>$ CISnj, and CISj $>$ CISnj.

\section{Juxtacortical Lesion Volume and Distribution, and Probability Maps}

Mean JLV was $1.28 \mathrm{~mL}$ (range, 0.03-14.14 mL) in patients with CIS, and $3.41 \mathrm{~mL}$ (range, $0.10-22.70 \mathrm{~mL}$ ) in those with RRMS. The absolute JLV was greater at the frontal lobe, followed by the parietal, temporal, and occipital lobe, and insula in both CIS and RRMS (Table 2). The calculation of JLV relative to the total volume of each lobe in patients with CIS showed that the insula had the highest volume of JLs per volume of lobe, followed by the temporal, parietal, frontal, and occipital lobes. The rank order for RRMS was insula, followed by temporal, frontal, parietal, and occipital (Table 2).

\section{Global and Lobar Cortical Thickness}

In general, the lowest cortical thickness was measured in patients with RRj, followed by CISj, RRnj, CISnj, and CISn at both global and lobar levels (Table 3). The percentage difference between CISn and RRj ranged from $0 \%$ (insula) to $4 \%-4.5 \%$ (occipital, temporal, and parietal lobes). Regions with the highest attenuation of JL do not correspond to the ones with the largest thickness loss. A visual comparison of the lobar cortical thickness loss (mean of the right and left hemispheres) in RRj compared with

Table 3: Global and lobar mean cortical thickness (in $\mathrm{mm}$ ) for the groups studied

\begin{tabular}{|c|c|c|c|c|c|c|c|}
\hline Lobe & $\begin{array}{c}P \text { Value } \\
\text { (Factor Group) }^{a}\end{array}$ & CISn & CISnj & CISj & RRnj & RRj & 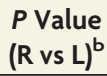 \\
\hline Global_R & .064 & $2.50(0.13)^{c}$ & $2.47(0.09)$ & $2.44(0.16)$ & $2.47(0.09)$ & $2.40(0.13)$ & .806 \\
\hline Global_L & .074 & $2.50(0.13)$ & $2.48(0.19)$ & $2.45(0.16)$ & $2.49(0.09)$ & $2.41(0.13)$ & \\
\hline Frontal_R & .389 & $2.55(0.16)$ & $2.53(0.16)$ & $2.51(0.16)$ & $2.55(0.11)$ & $2.49(0.11)$ & .006 \\
\hline Frontal_L & .424 & $2.52(0.17)$ & $2.47(0.11)$ & $2.46(0.15)$ & $2.50(0.13)$ & $2.46(0.12)$ & \\
\hline Insula_ $\bar{R}$ & .944 & $3.01(0.16)$ & $3.04(0.14)$ & $3.02(0.25)$ & $2.99(0.15)$ & $3.03(0.13)$ & .711 \\
\hline Insula_L & .881 & 3.05 (0.19) & $3.05(0.14)$ & $3.01(0.24)$ & $3.03(0.16)$ & $3.02(0.15)$ & \\
\hline Parietal_R & .056 & $2.22(0.11)^{\mathrm{c}}$ & $2.17(0.07)$ & $2.15(0.17)$ & $2.19(0.08)$ & $2.12(0.13)$ & .414 \\
\hline Parietal_L & .025 & $2.22(0.11)^{c}$ & $2.20(0.09)$ & $2.17(0.17)$ & $2.21(0.09)$ & $2.12(0.14)$ & \\
\hline Temporal_R & .119 & $2.93(0.14)$ & $2.90(0.17)$ & $2.87(0.21)$ & $2.91(0.21)$ & $2.80(0.21)$ & .002 \\
\hline Temporal_L & .084 & $3.01(0.14)$ & $2.97(0.16)$ & $2.94(0.20)$ & $2.96(0.16)$ & $2.88(0.19)$ & \\
\hline Occipital_R & .014 & $2.29(0.14)$ & $2.30(0.11)^{c}$ & $2.24(0.17)$ & $2.29(0.12)$ & $2.19(0.14)$ & .224 \\
\hline Occipital L & .047 & $2.30(0.13)$ & $2.31(0.09)^{c}$ & $2.28(0.13)$ & $2.29(0.11)$ & $2.22(0.15)$ & \\
\hline
\end{tabular}

Note:- R indicates right hemisphere; L, left hemisphere. Numbers in parentheses are the SD.

a $P$ value of the 1-way ANOVA test with group as a factor.

${ }^{b} P$ value of the hemisphere effect ( $R$ vs $L$ hemispheres values).

${ }^{c} P<.05$ compared with RRj. 


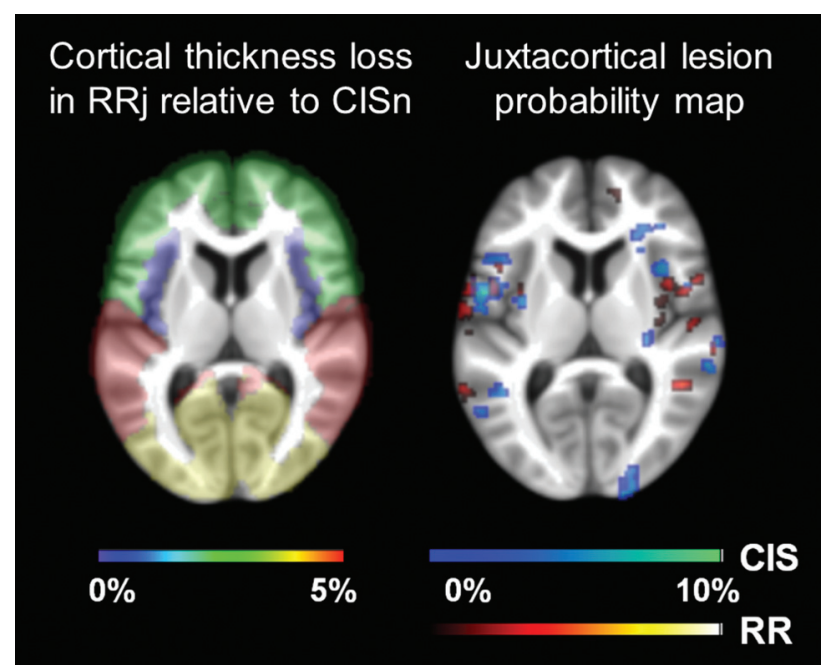

FIG 1. Percentage cortical thickness loss in RRj relative to CISn for the different brain lobes (frontal, insula, parietal, temporal, and occipital). Values were color-coded and ranged from $0 \%-5 \%$. Juxtacortical lesion probability map (right) for patients with CIS (cold scale) and patients with RRMS (warm scale), scaled to the maximum value (10\%). Results were overlaid on the mean filled structural image of the whole cohort studied.

CISnj and the JL probability map (of patients with CIS and patients with RRMS) can be seen in Fig 1.

On multivariate analysis of global values, the presence of JLs was significant $\left(P\right.$, adjusted $\left.R^{2}\right)$ in the right $(.004,0.166)$ and left $(.005,0.165)$ hemispheres, JLV was significant in the right hemisphere $(.033,0.303)$, and LV was significant in the left hemisphere $(.039,0.646)$. Lobar analysis showed that insular cortical thickness was not related with the presence of JLs, JLV, or LV, in either hemisphere. The $\left(P\right.$, adjusted $\left.R^{2}\right)$ right frontal $(.048,0.145 ; .043$, $0.639)$, right parietal $(.005,0.156 ; .020,0.696)$, and right occipital $(.000,0.157 ; .007,0.753)$ lobes showed a significant association with the presence of JLs or LV. JLV was significant in the right parietal $(.018,0.327)$ and right occipital $(.011,0.315)$ lobes. Left frontal cortical thickness was not associated with any of the factors; left temporal lobe thickness was associated with the presence of JLs $\left(P\right.$, adjusted $\left.R^{2}\right)(.009,0.114)$ and in left parietal $(.002$, $0.166 ; .035,0.649 ; .005,0.368)$ and left occipital lobules $(.004$, $0.146 ; .038,0.639 ; .001,0.417)$, thickness was associated with the 3 factors (presence JLs, LV, JLV).

\section{Regional Cortical Thickness}

Results at a regional level were along the same lines as the ones obtained at a global and lobar level, with the group RRj showing the thinnest cortical thickness, followed by CISj, RRnj, CISnj, and CISn. Significant (post hoc) comparisons indicate that CISn measured cortical thickness was greater compared with $\mathrm{RRj}$ in the following regions (by following FreeSurfer nomenclature): right cuneus, right entorhinal, right and left fusiform, right inferior parietal, right lateral occipital, right and left precuneus, and left paracentral. Another (post hoc) comparison that was significant was CISnj over RRj in the right cuneus, right lateral occipital, left isthmus cingulate, left paracentral, and left transverse temporal areas. In addition, RRj showed a significant cortical thinning compared with CISj in the left banks of superior temporal sulcus and left paracentral regions. Finally, cortical thickness for CISnj was significantly thinner compared with CISn at the right frontal pole.

\section{Cortical Thickness and Juxtacortical Lesion Location}

Only patients with JLs (CISj and RRj) were included in this subanalysis. The regions analyzed were those in which a JL had been detected in at least 9 patients: banks of superior temporal sulcus, fusiform, inferior temporal, insula, postcentral, rostral middle frontal, superior frontal, superior temporal, and temporal pole. In separate analyses of the CIS and RRMS groups, the mean cortical thickness in each selected region was compared between patients with and without JLs (2-sample $t$ test). In the RRMS group alone, a nonsignificant trend to decreased cortical thickness in the superior frontal region was seen in patients with JLs $(P=.079)$. Finally, to visually check whether changes in cortical thickness showed co-localization with JLs, a binary mask of the JL probability map was transferred to the inflated brain representation and overlaid onto the corresponding contrast (CISnj $>\mathrm{CISj}$ with the CIS map, and RRnj > RRj with the RRMS map). On visual inspection, cortical thickness changes were not found to co-localize with JL probability maps (Figs 2 and 3 ).

\section{Subcortical Gray Matter Volume Changes and Presence of Juxtacortical Lesions}

The estimated total intracranial volume did not differ between groups: mean $(\mathrm{SD}) \mathrm{CISn}=1337.73 \pm 189.85 \mathrm{~mL}$, CISnj $=$ $1331.71 \pm 192.74 \mathrm{~mL}, \mathrm{CISj}=1381.25 \pm 173.46 \mathrm{~mL}, \mathrm{RRnj}=$ $1363.95 \pm 190.06 \mathrm{~mL}$, and $\mathrm{RRj}=1392.59 \pm 184.77 \mathrm{~mL}$. Subcortical gray matter volumes in all the structures analyzed were lower in patients with $\mathrm{RRj}$ (Table 4). Volume loss ranged from $8 \%$ (caudate) to almost $20 \%$ (accumbens) when compared with CISnj. The largest right-to-left asymmetry was found in the pallidum (ratio, 0.87-0.92), followed by the putamen (0.94-0.98). The caudate was also significantly asymmetrical, though in the opposite direction: right-to-left ratio ranged between 1.03 and 1.06 .

On multivariate analysis, the presence of JLs was a significant variable in the right thalamus ( $P$, adjusted $\left.R^{2}\right)(.000,0.130)$, left thalamus $(.001,0.120)$, right caudate $(.000,0.135)$, left caudate $(.000,0.152)$, right putamen $(.000,0.162)$, left putamen $(.000$, $0.147)$, left pallidum $(.000,0.145)$, right hippocampus $(.020$, $0.124)$, left hippocampus $(.046,0.127)$, right amygdala $(.004,0.081)$, right accumbens $(.000,0.171)$ and left accumbens $(.000,0.120) ; \mathrm{JLV}$ and LV did not reach significance in any region.

\section{DISCUSSION}

This study investigated, for the first time, the association between the presence and topography of JLs and cortical thinning, and subcortical gray matter volume measurements in patients with CIS and patients with RRMS.

Patients with RRMS had a larger number of JLs than patients with CIS. In the cohort studied, the JL volume in patients with RRMS was twice the volume seen in patients with CIS, but JL brain distribution was similar in the 2 patient populations. Most JLs were located in the frontal lobe, followed by the parietal, temporal, and occipital lobes, a distribution that is similar to the reported distribution of pure cortical lesions in RRMS. ${ }^{24,25}$ When 


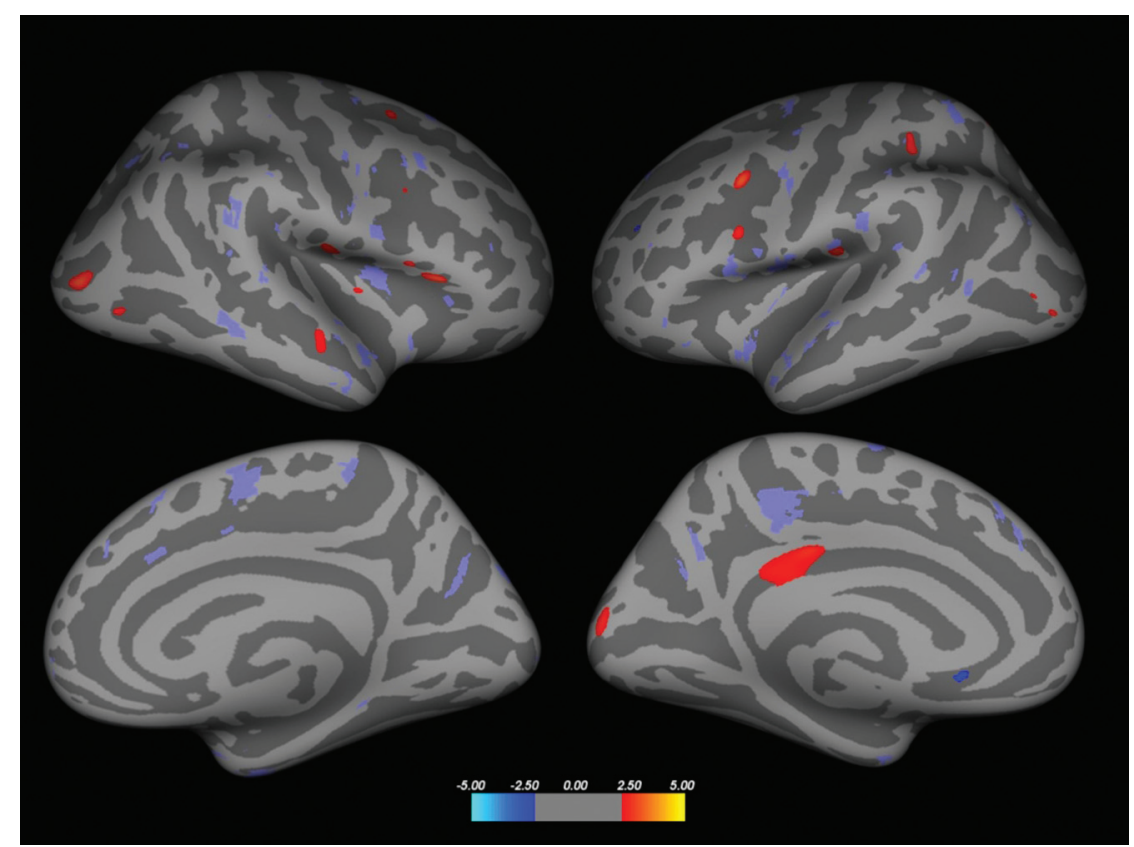

FIG 2. Inflated brain, displaying areas of reduced cortical thickness (red areas) in CISj compared with CISnj (significance level $P<.01$ for display purposes). Blue areas indicate regions where cortical thickness in CISnj is reduced compared with CISj. The CIS JL lesion probability map was overlaid in purple (right column, left hemisphere; left column, right hemisphere; superior row, lateral view; inferior row, medial view). The JL lesion probability map is a binary mask for display purposes. No overlap was seen between the areas, showing significant changes in thickness and the JL lesion probability map.

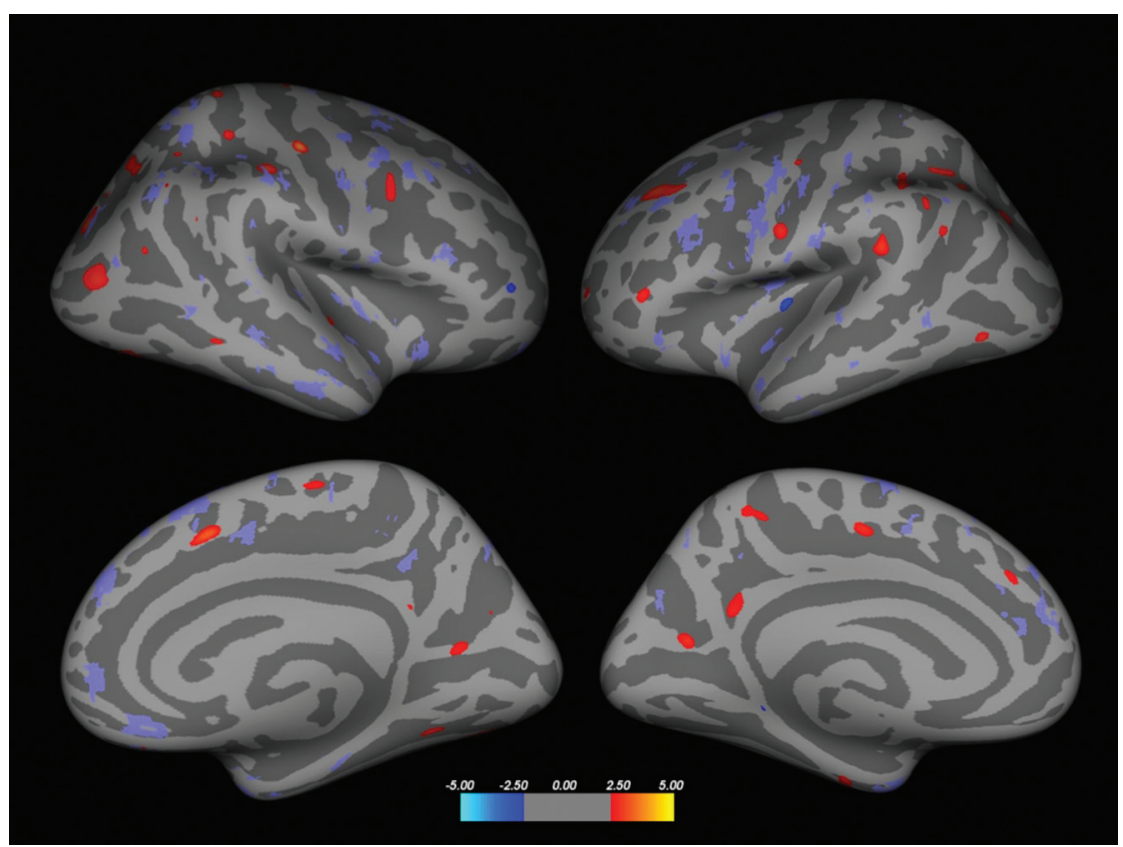

FIG 3. Inflated brain displaying areas of reduced cortical thickness (red areas) in RRj compared with RRnj (significance level $\mathrm{P}<.01$, for display purposes). Blue areas indicate regions in which cortical thickness in RRnj is reduced compared with RRj. The RR JL lesion probability map was overlaid in purple (right column, left hemisphere; left column, right hemisphere; superior row, lateral view; inferior row, medial view). The JL lesion probability map was a binary mask, just for display purposes. No overlap was seen between the areas showing significant changes in thickness and the JL lesion probability map.

JLVs were normalized by the lobe volume, the insula appeared to be the region with the highest attenuation of JLs, followed by the temporal, parietal, and frontal lobes. The LV was higher in pa- tients with RRj, but the value in RRnj was even lower than in CISj, though the differences were not significant. These results may indicate that $\mathrm{JL}$ accumulation is not disconnected from accumulation of lesions in other areas of the brain and that they both are part of the same disease process regardless of disease stage.

The RRj group was found to have the greatest global and regional cortical thinning in areas of the temporal, parietal, and occipital lobe. The most marked thickness loss (RRj compared with CISn) was in the right entorhinal cortex $(8 \%)$, in line with the findings of Narayana et al. ${ }^{26}$ Entorhinal thinning is considered a predictor of cognitive decline in Alzheimer disease. ${ }^{27}$ Our study did not include a neuropsychological evaluation; therefore, we could not analyze the value of entorhinal atrophy for predicting cognitive decline in our patients with MS. When following the entorhinal cortex, we found greater thinning at the right precuneus and cuneus $(6 \%)$. Decreases in the remaining regions of the right brain hemisphere and all regions of the left brain hemisphere ranged from $3 \%$ to $5 \%$. The precuneus and fusiform were the only regions that showed bilateral cortical thinning.

Our results also indicated that cortical thinning was more closely associated with the presence of JLs than with total LV or JL volume, though LV was $<10$ $\mathrm{mL}$ in $90 \%$ of patients. Results of studies reported to date, including LV as a variable, are not conclusive because the threshold value used to define high and low LV has not been established univocally. ${ }^{12,14}$ In a previous study by Charil et al, ${ }^{17}$ the frontal, insula, and temporal regions showed the most marked cortical thinning, and cortical atrophy correlated with lesion load.

Visual inspection of JL probability maps overlaid onto the cortical change maps of the 2 hemispheres showed no overlapping in either patients with CIS or those with RRMS. A more detailed subanalysis of this aspect confirmed that differences in cortical thickness were not directly related to the presence of JLs in any specific region, which indicates that cortical thinning in MS is not closely dependent on the topography of JLs. This discrepancy may arise from the fact that only a percentage of JLs and a minority of intracortical lesions are visible 
Table 4: Subcortical gray matter volumes percentage relative to total intracranial volume for the groups studied

\begin{tabular}{|c|c|c|c|c|c|c|c|}
\hline $\begin{array}{c}\text { Subcortical Gray } \\
\text { Matter }\end{array}$ & $\begin{array}{c}P \text { Value } \\
\text { (Factor Group) }^{\text {a }}\end{array}$ & $\mathrm{CISn}^{\mathrm{g}}$ & $\mathrm{CISnj}^{\mathrm{g}}$ & $\mathrm{CISj}^{\mathrm{g}}$ & $R_{R n j}{ }^{g}$ & $R_{R j}{ }^{g}$ & $\begin{array}{l}P \text { Value } \\
(R \text { vs } L)^{b}\end{array}$ \\
\hline Thal_R & .000 & $0.502(0.041)^{f}$ & $0.486(0.060)^{\mathrm{e}}$ & $0.473(0.058)^{\mathrm{e}}$ & $0.477(0.092)$ & $0.424(0.064)$ & .986 \\
\hline Thal_L & .003 & $0.492(0.042)^{\mathrm{e}}$ & $0.485(0.053)^{e}$ & $0.474(0.062)$ & $0.476(0.010)$ & $0.432(0.066)$ & \\
\hline Pallidum R & .029 & $0.115(0.013)^{c}$ & $0.114(0.016)^{d}$ & $0.109(0.015)$ & $0.110(0.017)$ & $0.103(0.017)$ & .000 \\
\hline Pallidum_L & .003 & $0.132(0.018)^{\mathrm{e}}$ & $0.129(0.017)^{d}$ & $0.118(0.023)$ & $0.124(0.027)$ & $0.113(0.021)$ & \\
\hline Caudate_R & .069 & $0.277(0.037)$ & $0.278(0.036)$ & $0.257(0.041)$ & $0.271(0.052)$ & $0.256(0.035)$ & .015 \\
\hline Caudate_L & .025 & $0.268(0.036)$ & $0.266(0.034)$ & $0.247(0.037)$ & $0.259(0.043)$ & $0.242(0.038)$ & \\
\hline Putamen_R & .009 & $0.428(0.037)^{d}$ & $0.427(0.074)^{d}$ & $0.408(0.072)$ & $0.410(0.085)$ & $0.369(0.072)$ & .020 \\
\hline Putamen_L & .002 & $0.452(0.042)^{\mathrm{e}}$ & $0.452(0.069)^{\mathrm{e}}$ & $0.428(0.081)$ & $0.430(0.082)$ & $0.384(0.073)$ & \\
\hline Accum_ $\mathrm{R}^{-}$ & .001 & $0.054(0.011)^{d}$ & $0.056(0.011)^{e}$ & $0.052(0.009)$ & $0.057(0.012)^{d}$ & $0.045(0.013)$ & .745 \\
\hline Accum_L & .002 & $0.053(0.007)$ & $0.058(0.012)^{\mathrm{e}}$ & $0.052(0.009)$ & $0.054(0.010)$ & $0.047(0.010)$ & \\
\hline Hippo_R & .064 & $0.305(0.026)$ & $0.315(0.041)^{d}$ & $0.306(0.040)$ & $0.306(0.053)$ & $0.283(0.053)$ & .393 \\
\hline Hippo_L & .121 & $0.301(0.026)$ & $0.308(0.041)$ & $0.303(0.037)$ & $0.294(0.056)$ & $0.282(0.044)$ & \\
\hline Amygdala_R & .005 & $0.129(0.017)$ & $0.140(0.023)^{\mathrm{e}}$ & $0.128(0.020)$ & $0.131(0.025)$ & $0.119(0.024)$ & .440 \\
\hline Amygdala_L & .158 & $0.126(0.020)$ & $0.134(0.021)$ & $0.129(0.019)$ & $0.128(0.021)$ & $0.120(0.028)$ & \\
\hline
\end{tabular}

Note:-Thal indicates thalamus; R, right; L, left; Accum, accumbens; Hippo, hippocampus.

a $P$ value of the 1-way ANOVA test with group as a factor.

${ }^{\mathrm{b}} P$ value of the hemisphere effect ( $R$ vs $L$ hemispheres values).

${ }^{c} P=.059$

d $p<.05$.

e $p<.01$.

${ }^{f} P<.001$ compared with RRj.

${ }^{g}$ Data are expressed as mean (SD)

on conventional MR imaging (mainly because of their size), though those that are seen highly correlate with the overall number of cortical lesions detected on a histopathologic study. ${ }^{27} \mathrm{~A}$ study published by Bakshi et $\mathrm{al}^{28}$ found the there was a correlation between the number of cortical and juxtacortical lesions for a given region and the cortical atrophy measured in that region. Discrepancy may arise again from the fact that we evaluated the presence and volume (not the number) of juxtacortical lesions, which actually represent a small percentage compared with cortical lesions.

In the present study, the volume of subcortical gray matter structures was systematically smaller in patients with RRj than in patients with CISn. Although the volume of subcortical gray matter structures in RRnj was systematically larger than in CISj, this post hoc comparison was not significant in any of the structures analyzed. A recent study that compared subcortical gray matter volumes between CIS and early RRMS $^{14}$ also showed that early RRMS volumes were smaller compared with CIS and that differences were significant in some regions (caudate, putamen, thalamus) but not in others (amygdala, accumbens); this was in contrast with the absence of differences in cortical structures. However, these findings were not stratified by the presence of cortical lesions or JL. In this regard, results from our multivariate analysis indicated that the presence of JL was associated with the volume of a number of subcortical structures, whereas JLV and LV were not.

Taken together, the results reported here would indicate that the presence of JL could be used as a marker of diffuse gray matter damage; it could be speculated that the appearance of JLs is the by-product of the damaging pathologic process ongoing in gray matter of people with MS and not the other way around (JLs and locally related cortical lesions being the main cause of damage to gray matter). This hypothesis should be tested in future studies.

The limitations of our study include the small size of the RRnj group; this is because few patients with RRMS exclusively have lesions other than JLs. Another limitation is that the presence of both JL and JLV were colinear with the LV, and we were not able to add the
LV as a covariate in the statistical analysis. The lack of a control group of healthy subjects was also a limitation of the study because comparisons with normative values would be of great interest. Further studies with a larger group and a paired control group would be needed to confirm the main findings of our work. In addition, identification of JLs was based on visual inspection by using conventional T2-FLAIR sequences, which may have underestimated the number of these lesions. The partial volume effect could also play a role, which affected the visualization of the lesions in the axial images. Cortical lesions are abundantly present in MS and are better detected with dedicated pulse sequences, such as double inversion recovery, phase-sensitive inversion recovery, and high-resolution 3D MPRAGE. ${ }^{3,29-34}$ Imaging of cortical lesions at standard clinical field strength remains suboptimal even when combinations of these sequences (double inversion recovery, phase-sensitive inversion recovery, and MPRAGE) are used because of limitations in the associated sensitivity and reproducibility. ${ }^{35,36}$ Thus, although these sequences have made a major contribution in detecting focal cortical lesions in patients with MS and have provided important insights about cortical abnormalities and their association with clinical disability and cognitive impairment in all MS subtypes, substantial research efforts are needed before they can be used in the diagnostic imaging work-up in clinical practice. Finally, the local and individual effect of brain juxtacortical lesions on cortical thickness measurements should also be closely studied.

\section{CONCLUSIONS}

Cortical thinning and subcortical gray matter volume loss in patients with CIS or RRMS was related to the presence of JLs, though the cortical areas with the most marked thinning did not correspond to those with the largest number of JLs, which may indicate that visible focal JLs do not completely explain, but might be used as markers of the diffuse gray matter damage that is already present in the early phases of MS.

AJNR Am J Neuroradiol 36:2270-76 Dec 2015 www.ajnr.org 


\section{ACKNOWLEDGMENTS}

We thank the "Red Española de Esclerosis Múltiple)" (RD07/ 0060; RD12/0032), which is sponsored by the Fondo de Investigación Sanitaria, the Instituto de Salud Carlos III, the Ministry of Economy and Competitiveness in Spain, and the "Ajuts per donar Suport als Grups de Recerca de Catalunya (2009 SGR 0793)," which is sponsored by the "Agència de Gestió d'Ajuts Universitaris i de Recerca" of the Generalitat de Catalunya in Spain.

Disclosures: Jaume Sastre-Garriga—UNRELATED: Consultancy: Almirall; Grants/ Grants Pending: Genzyme; Payment for Lectures (including service on speakers bureaus): Genzyme, Merck-Serono, Novartis, Teva, Biogen; Payment for Development of Educational Presentations: Novartis; Travel/Accommodations/Meeting Expenses Unrelated to Activities Listed: Novartis. Cristina Auger-UNRELATED: Payment for Lectures (including service on speakers bureaus): Novartis. Mar TintoréUNRELATED: Board Membership: Biogen, Genzyme, TEVA; Grants/Grants Pending: Biogen, ${ }^{*}$ Novartis*; Payment for Lectures (including service on speakers bureaus): Biogen, Genzyme, TEVA, Novartis, Merk, Bayer. Xavier Montalban—UNRELATED: Consultancy: Actelion, Almirall, Bayer, Biogen Idec, Genzyme, GlaxoSmithKline, Merck, Novartis, Sanofi-Genzyme, Roche, Teva. Alex Rovira-UNRELATED: Board Membership: Genzyme, Novartis, Biogen, OLEA; Payment for Lectures (including service on speakers bureaus): Novartis, Stendhal, Biogen, Genzyme, Olea, Bracco, Bayer. *Money paid to the institution.

\section{REFERENCES}

1. Filippi M, Rocca MA, Barkhof F, et al. Association between pathological and MRI findings in multiple sclerosis. Lancet Neurol 2012; 11:349-60 CrossRef Medline

2. Kutzelnigg A, Lucchinetti CF, Stadelmann C, et al. Cortical demyelination and diffuse white matter injury in multiple sclerosis. Brain 2005;128:2705-12 CrossRef Medline

3. Geurts JJ, Pouwels PJ, Uitdehaag BM, et al. Intracortical lesions in multiple sclerosis: improved detection with 3D double inversion-recovery MR imaging. Radiology 2005;236:254-60 CrossRef Medline

4. Geurts JJ, Blezer EL, Vrenken H, et al. Does high-field MR imaging improve cortical lesion detection in multiple sclerosis? J Neurol 2008;255:183-91 CrossRef Medline

5. Wegner C, Esiri MM, Chance SA, et al. Neocortical neuronal, synaptic, and glial loss in multiple sclerosis. Neurology 2006:67;960-67 CrossRef

6. Calabrese M, Favaretto A, Martini V, et al. Grey matter lesions in MS: from histology to clinical implications. Prion 2013;7:20-7 CrossRef Medline

7. Roosendaal SD, Moraal B, Pouwels PJ, et al. Accumulation of cortical lesions in MS: relation with cognitive impairment. Mult Scler 2009; 15:708-14 CrossRef Medline

8. Calabrese M, Rocca MA, Atzori M, et al. A 3-year magnetic resonance imaging study of cortical lesions in relapse-onset multiple sclerosis. Ann Neurol 2010;67:376-83 CrossRef Medline

9. Calabrese M, Favaretto A, Poretto V, et al. Low degree of cortical pathology is associated with benign course of multiple sclerosis. Mult Scler 2013;19:904-11 CrossRef Medline

10. Filippi M, Rocca MA, Horsfield MA, et al. Imaging cortical damage and dysfunction in multiple sclerosis. JAMA Neurol 2013;70: 556-64 CrossRef Medline

11. Audoin B, Zaaraoui W, Reuter F, et al. Atrophy mainly affects the limbic system and the deep grey matter at the first stage of multiple sclerosis. J Neurol Neurosurg Psychiatry 2010;81:690-95 CrossRef Medline

12. Sailer M, Fischl B, Salat D, et al. Focal thinning of the cerebral cortex in multiple sclerosis. Brain 2003;126:1734-44 CrossRef Medline

13. Calabrese M, Rinaldi F, Mattisi I, et al. The predictive value of gray matter atrophy in clinically isolated syndromes. Neurology 2011;77: 257-63 CrossRef Medline

14. Bergsland N, Horakova D, Dwyer MG, et al. Subcortical and cortical gray matter atrophy in a large sample of patients with clinically isolated syndrome and early relapsing-remitting multiple sclerosis. AJNR Am J Neuroradiol 2012;33:1573-78 CrossRef Medline
15. Bermel RA, Bakshi R. The measurement and clinical relevance of brain atrophy in multiple sclerosis. Lancet Neurol 2006;5:158-70 CrossRef Medline

16. Ramasamy DP, Benedict RH, Cox JL, et al. Extent of cerebellum, subcortical and cortical atrophy in patients with MS: a case-control study. J Neurol Sci 2009;282:47-54 CrossRef Medline

17. Charil A, Dagher A, Lerch JP, et al. Focal cortical atrophy in multiple sclerosis: relation to lesion load and disability. Neuroimage 2007;34: 509-17 CrossRef Medline

18. Miller DH, Chard DT, Ciccarelli O. Clinically isolated syndromes. Lancet Neurol 2012;11:157-69 CrossRef Medline

19. Tzourio-Mazoyer N, Landeau B, Papathanassiou D, et al. Automated anatomical labeling of activations in SPM using a macroscopic anatomical parcellation of the MNI MRI single-subject brain. Neuroimage 2002;15:273-89 CrossRef Medline

20. Schmidt P, Gaser C, Arsic M, et al. An automated tool for detection of FLAIR-hyperintense white-matter lesions in multiple sclerosis. Neuroimage 2012;59:3774-83 CrossRef Medline

21. Pareto D, Sastre-Garriga J, Tintore M, et al. Grey and white matter segmentation in multiple sclerosis patients: correcting lesion misclassification with the lesion segmentation toolbox (LST). Mult Scler 2013;19:(S1)410

22. Dale AM, Fischl B, Sereno MI. Cortical surface-based analysis. I. Segmentation and surface reconstruction. Neuroimage 1999;9: 179-94 CrossRef Medline

23. Fischl B, Dale AM. Measuring the thickness of the human cerebral cortex from magnetic resonance images. Proc Natl Acad Sci U S A 2000;97:11050-55 CrossRef Medline

24. Battaglini M, Calabrese M, Stromillo ML, et al. Similar cortical lesion distribution and cortical atrophy location in patients with relapsing-remitting multiple sclerosis. Mult Scler 2010;16:S197-352

25. Calabrese $M$, Battaglini $M$, Giorgio A, et al. Imaging distribution and frequency of cortical lesions in patients with multiple sclerosis. Neurology 2010;75:1234-40 CrossRef Medline

26. Narayana PA, Govindarajan KA, Goel P, et al. Regional cortical thickness in relapsing remitting multiple sclerosis: a multi-center study Neuroimage Clin 2012;2:120-31 CrossRef Medline

27. Velayudhan L, Proitsi P, Westman E, et al. Entorhinal cortex thickness predicts cognitive decline in Alzheimer's disease. J Alzheimers Dis 2013;33:755-66 CrossRef Medline

28. Bakshi R, Ariyaratana S, Benedict RH, et al. Fluid-attenuated inversion recovery magnetic resonance imaging detects cortical and juxtacortical multiple sclerosis lesions. Arch Neurol 2001;58:742-48 Medline

29. Seewann A, Vrenken H, Kooi EJ, et al. Imaging the tip of the iceberg: visualization of cortical lesions in multiple sclerosis. Mult Scler 2011;17:1202-10 CrossRef Medline

30. Sethi V, Yousry TA, Muhlert N, et al. Improved detection of cortical MS lesions with phase-sensitive inversion recovery MRI. J Neurol Neurosurg Psychiatry 2012;83:877-82 CrossRef Medline

31. Calabrese M, De Stefano N, Atzori M, et al. Detection of cortical inflammatory lesions by double inversion recovery magnetic resonance imaging in patients with multiple sclerosis. Arch Neurol 2007; 64:1416-22 CrossRef Medline

32. Wattjes MP, Lutterbey GG, Gieseke J, et al. Double inversion recovery brain imaging at $3 \mathrm{~T}$ : diagnostic value in the detection of multiple sclerosis lesions. AJNR Am J Neuroradiol 2007;28:54-59 Medline

33. Simon B, Schmidt S, Lukas C, et al. Improved in vivo detection of cortical lesions in multiple sclerosis using double inversion recovery $M R$ imaging at 3 Tesla. Eur Radiol 2010;20:1675-83 CrossRef Medline

34. Nelson F, Poonawalla A, Hou P, et al. 3D MPRAGE improves classification of cortical lesions in multiple sclerosis. Mult Scler 2008;14: 1214-19 CrossRef Medline

35. Geurts JJ, Roosendaal SD, Calabrese M, et al. Consensus recommendations for MS cortical lesion scoring using double inversion recovery MRI. Neurology 2011;76:418-24 CrossRef Medline

36. Sethi V, Muhlert N, Ron M, et al. MS cortical lesions on DIR: not quite what they seem? PLoS One 2013;8:e78879 CrossRef Medline 